\title{
Worst Case Braking Trajectories for Robotic Motion Simulators
}

\author{
Andreas Labusch $^{\dagger}$, Tobias Bellmann ${ }^{\dagger}$, Karan Sharma ${ }^{\ddagger}$, Johann Bals ${ }^{\dagger}$
}

\begin{abstract}
Motion simulators based on industrial robots can produce high dynamic accelerations and velocities compared to classical hydraulic hexapod systems. In case of emergency stops, large and possibly harmful accelerations can occur. This paper aims to provide an optimization procedure to generate worst case trajectories in order to test for these harmful accelerations, by maximizing the kinetic energy prior the emergency stop. The dynamical and mechanical limits of the robot are considered as constraints of the optimization criterion. An exemplary worst case trajectory is simulated using a braking model and the resulting Head Injury Criterion (HIC) is calculated and compared with older tests, using non-optimized trajectories. A significant higher, yet with the current robot dynamics not harmful HIC value can be generated.
\end{abstract}

\section{INTRODUCTION}

Motion simulators based on serial kinematics have lately come into focus of research [1], [2], [3] and commercial use as flight simulators ${ }^{1}$. The safety of the pilot is generally ensured by several certified safety systems, as for instance lowlevel surveillance through robot control, safety precautions in path planning, limited joint angles preventing self-collisions and hardware stops in case of a fatal structural failure.

Nevertheless, during operation of such a robotic motion platform, software errors can occur, making an emergency stop necessary, for example missing motion data due to network errors or power failure [4]. Recently we investigated the accelerations [5], [6] caused by such emergency stops from high-speed robot trajectories using an anthropomorphic test device (also called "crash test dummy"). We showed that the resulting movements are harmless according to the Head Injury Criterion (HIC) [7], [8] and Neck Injury Criterion (NIC) [7].

Although we found the resulting accelerations being far below the threshold for neck and head injuries, the tested trajectories for evaluating the HIC have been selected by maximizing only the velocities of axes working in the same direction, e.g. Axis 2, 3 and 5 of the used robot (DLR Robotic Motion Simulator, see figure 1), followed by triggering the emergency stop when reaching the highest joint velocities. Most studies concerning the safety of humans in Human-Robot systems consider humans to be in the immediate vicinity of the robotic systems. In the RMS, in contrast, the pilot is part of the robot system.

In this paper we present a method to deduce a worst case

This work is supported by KUKA Roboter GmbH

${ }^{\dagger}$ Institute of System Dynamics and Control - German Aerospace Center (DLR), 82234 Oberpfaffenhofen, Germany

\#Institute of Robotics and Mechatronics - German Aerospace Center (DLR) , 82234 Oberpfaffenhofen, Germany

Contact: andreas.labusch@dlr.de

${ }^{1}$ http://www.grenzebach.com/index.php/grenzebach/technologien/flightsim

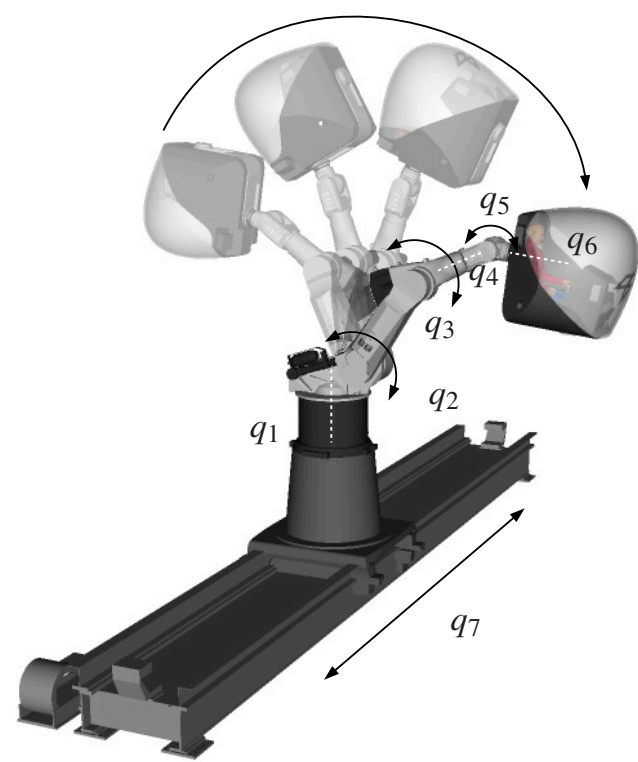

Fig. 1. The DLR Robotic Motion Simulator with its 7 axes $q_{1}, \ldots, q_{7}$, performing a whiplash trajectory using the axes 2, 3 and 5 . This maneuver has been investigated with a crash test dummy in [6] and is used as a handselected reference trajectory for comparison with the optimized worst case trajectory in this paper.

trajectory by optimizing the maximum kinetic energy of the passenger's head, considering the mechanical and dynamical constraints of the robot arm as well as the workspace needed to accelerate the arm to the necessary joint velocities.

\section{METHODS}

A worst case braking trajectory (WCBT) is defined as a robot motion, designed to increase the danger of the pilot getting injured by emergency braking. To quantify the danger for the pilot, we are using the HIC but it is also possible to consider other kinds of criteria [7]. As per [9] the HIC is defined as follows:

$$
\operatorname{HIC}\left(\Delta t_{\max }\right)=\max _{\Delta t}\left[\Delta t\left(\frac{1}{\Delta t} \int_{t_{1}}^{t_{2}}\|\hat{a}\| \mathrm{d} t\right)^{5 / 2}\right] .
$$

The scaled acceleration of the head, with respect to $g$, $\hat{a}=a / g, g=9.81 \mathrm{~m} / \mathrm{s}^{2}$, is integrated from $t_{1}$ to $t_{2}$, over the time period $\Delta t=t_{2}-t_{1} \leq t_{\max }$. The acceleration of the head $a$ is the second derivative of the position and does not take the gravity into account. The considered time frame $t_{\max }$ is $15 \mathrm{~ms}$ for $\mathrm{HIC}_{15}$ or $36 \mathrm{~ms}$ for $\mathrm{HIC}_{36}$. The start time $t_{1}$ is varied around the peak to maximize the resulting value. 
This criterion is used e.g. for head injury analysis in car crashes by EuroNCAP [10].

The NIC rather takes the spine into account. It can be calculated by

$$
\mathrm{NIC}=0.2 \cdot a_{r e l}+v_{r e l}^{2}
$$

where $a_{r e l}$ is the relative acceleration between the first spinal vertebra and first thoracic vertebra and $v_{r e l}$ is the time integral of $a_{\text {rel }}$ [11].

These two criteria show the important influence of the duration and amount of acceleration. As done in other studies [8] we want to increase the kinetic energy to get an intensive and long braking acceleration. Because of the workspace limitations of the robot and its inhomogeneous capabilities of acceleration, the solution for optimal configurations is not obvious. In the literature review we undertook, we found no comparable work considering worst case trajectory generation for industrial robots.

\section{A. Structure of the DLR Robotic Motion Simulator}

The DLR Robotic Motion Simulator is an interactive motion simulator based on a six-axis industrial robot arm. The used robot (The DLR Robotic Motion Simulator, based on KUKA KR500-2 TÜV, see figure 1) is certified to transport humans safely by mechanical and software limitations. At the tool center point (TCP), a simulator cell is mounted sitting the pilot. The setup inside the cell includes two stereo projectors, exchangeable instruments, input devices like a steering wheel or a side stick. Together with the motion cues of the robot, they generate a virtual reality environment providing the pilot with an immersive simulation experience [4].

The robot is mounted on an additional linear axis increasing the workspace and allowing redundancy for movements.

For the presented method we derive the rigid dynamic equations of the used industrial robot arm. These can be formulated through the Newton-Euler approach or via Lagrange[12]: Let $\underline{q}$ be the joint angles of each joint, $\mathbf{M}$ the mass matrix of the system and $\underline{h}(\underline{q}, \dot{q})$ the vector including Coriolis and frictional forces. Additionally, the robot has a hydraulic counterbalance cylinder at the second axis, reducing the necessary motor torque. This unit is modeled and included in $\underline{h}(\underline{q}, \underline{\dot{q}})$. The vector $\underline{g}(\underline{q})$ describes gravitational effects. The required motor torques $\underline{\tau}$ of each axis can be calculated by

$$
\mathbf{M}(\underline{q}) \underline{\ddot{q}}+\underline{h}(\underline{q}, \underline{\dot{q}})+\underline{g}(\underline{q})=\underline{\tau} .
$$

These dynamic equations are modeled in Modelica $^{2}$ and have been verified for simulation purposes.

The configuration of the robot can be described by $q$ and $\dot{q}$. Figure 1 shows the robot with its joints numbered from $\overline{1}$ to 7 .

\footnotetext{
${ }^{2}$ Modelica and the Modelica Association, https://www.modelica.org/
}

\section{B. Maximizing the Kinetic Energy}

The HIC only takes translational accelerations into account. Maximizing the HIC means to optimize a trajectory for a 7 DOF system including the critical transition when the brakes are applied. Performing such a trajectory optimization for every possible path in the workspace requires a vast amount of computational time. Instead, we will maximize the translational kinetic energy in a single configuration with respect to necessary constraints which will be outlined. The translational kinetic energy of the pilot's head can be computed by:

$$
\begin{aligned}
E_{\text {kin, head }} & =\frac{1}{2} m_{\text {head }} v_{\text {head }}^{2} \\
& =\frac{1}{2} m_{\text {head }} \underline{\dot{\underline{r}}}_{\text {head }}^{T} \dot{\underline{r}}_{\text {head }} \\
& =\frac{1}{2} m_{\text {head }} \underline{\dot{q}}^{T} \mathbf{J}_{\text {head }}(\underline{q})^{T} \mathbf{J}_{\text {head }}(\underline{q}) \underline{\dot{q}},
\end{aligned}
$$

where $\mathbf{J}_{\text {head }}$ is the translational Jacobian matrix of the direct kinematics $\underline{f}_{\operatorname{dir}, r}(\underline{q})$ from the position of the robot base to the position of the head $\underline{r}_{\text {head }}$ :

$$
\mathbf{J}_{\text {head }}(\underline{q})=\frac{\partial \underline{f}_{\operatorname{dir}, r}(\underline{q})}{\partial \underline{q}}, \quad \underline{r}_{h e a d}=\underline{f}_{\operatorname{dir}, r}(\underline{q}) .
$$

To achieve maximum kinetic energy of the head, we generate an optimal trajectory that is followed by braking. The configuration at the end of the trajectory, that is also the beginning of the braking, is given by $\underline{q}_{e}$ and $\underline{\dot{q}}_{e}$.

Thus we define the optimization criterion which reduces to the norm of translational velocity of the head:

$$
\max _{\underline{q}_{e}, \underline{\dot{q}}_{e}}\left(\underline{\dot{q}}_{e}^{T} \mathbf{J}_{\text {head }}^{T}\left(\underline{q_{e}}\right) \mathbf{J}_{\text {head }}\left(\underline{q_{e}}\right) \underline{\dot{q}_{e}}\right),
$$

subject to the constrained joint angles $\underline{q} \min , \alpha, \underline{q}_{\max , \alpha}$ to prevent a collision with the hardware stops,

$$
\underline{q}_{\min , \alpha} \leq \underline{q}_{e} \leq \underline{q}_{\max }, \alpha,
$$

with

$$
\begin{aligned}
& \underline{q}_{\min , \alpha}=\underline{q}_{\min }+\alpha\left(\underline{q}_{\max }-\underline{q}_{\min }\right), \\
& \underline{q}_{\max , \alpha}=\underline{q}_{\max }-\alpha\left(\underline{q}_{\max }-\underline{q}_{\min }\right) .
\end{aligned}
$$

Further constraints are given through the robot control restricting the joint velocities and accelerations,

$$
\begin{aligned}
& -\beta \underline{\dot{q}}_{\text {max }} \leq \underline{\dot{q}}_{e} \leq \beta \underline{\dot{q}}_{\text {max }} \\
& -\gamma \underline{\ddot{q}}_{\text {max }} \leq \underline{\ddot{q}}_{e} \leq \gamma \underline{\ddot{q}}_{\text {max }} .
\end{aligned}
$$

The limits can be modified with $\alpha, \beta$ and $\gamma$ to simulate different configuration settings and to leave a safety gap that the braking does not end in any hardware stop:

$$
0 \leq \alpha, \beta, \gamma \leq 1
$$

Furthermore the torque $\underline{\tau}_{e}$ of each motor has to remain inside its limits,

$$
-\underline{\tau}_{\max } \leq \underline{\tau}_{e} \leq \underline{\tau}_{\max } .
$$

For a feasible solution to the optimization problem it is additionally necessary that the end configuration can be 
reached. Therefore we are assuming joint-wise maximum acceleration and calculating the starting position for each joint. The time to reach the end velocity is

$$
\underline{t}_{e}=\frac{\left\|\underline{\dot{q}}_{e}\right\|}{\gamma \underline{\underline{q}}_{\max }}
$$

thus the starting position $\underline{q}_{s}$ is given by

$$
q_{s, i}=q_{e, i}-\frac{1}{2} \gamma \operatorname{sgn}\left(\dot{q}_{e, i}\right) \ddot{q}_{\max , i} t_{e, i}^{2} \quad \forall i
$$

which leads to another constraint

$$
\underline{q}_{\min } \leq \underline{q}_{s} \leq \underline{q}_{\max } .
$$

The starting positions of the joints are only limited by the workspace of the robot and do not have a safety limit like the end positions. For the starting velocities we are defining

$$
\underline{\dot{q}}_{s}=\underline{0} .
$$

\section{Finding a Trajectory}

To reduce the complexity of the optimization procedure, we are only calculating end configurations with maximum kinetic energy. The trajectory to reach these configurations has to be reverse calculated from the optimized end position prior to the emergency stop. Fulfilling the constraints of maximum velocities, accelerations and the limits of the joint angles for the whole trajectory are implicitly granted due to the constraints (9) (12) (13). To ensure that the motor torques are not exceeded during acceleration, they are simulated using the rigid dynamics model (3). As each axis has different maximum acceleration and velocity and thus, a different start angle (17), the durations of the acceleration phases vary between the axes. This leads to different starting times $\underline{t}_{s}$ for each joint:

$$
t_{s, i}=t_{\max }-t_{e, i} \quad \forall i
$$

with $t_{\max }$ being the longest acceleration time of the slowest axis:

$$
t_{\max }=\max \left(t_{e, i}\right) \quad \forall i .
$$

Each joint accelerates with its maximum possible acceleration from $t_{s, i}$ to $t_{\max }$. To ensure that the calculated trajectory does not exceed the motor torques, the resulting motor torques are simulated and compared to their limits. If the motor torques violate their constraints during the trajectory, the result of the optimization is discarded as unfeasible and the constraints of the optimization criterion are reduced using the parameter $\gamma$. This extends the acceleration distance to the optimized end position but reduces the motor torques.

\section{Braking Model}

To simulate the accelerations of the emergency stop, a simple braking model of the axes has been implemented. The KUKA robot control provides three different braking modes [13]. "STOP 2" does normal deceleration through motor torques without brakes. In "STOP 1" the drives decelerate for one second and after that the brakes are closed and the drives are detached. With "STOP 0" the brakes are immediately closed and drives become unpowered. To determine the braking torques for simulation, former braking tests and data sheet information are used to calculate the applied torques of the brakes.

Each joint of the derived braking model consists of a drive, a clutch and a brake. Figure 2 shows the implementation of the power train to the rigid dynamics. While driving the trajectory the clutch is closed and the brakes are left open. With the brakes activated, the clutches are released to decouple the drives from the power train. The inertia of the motors are added to the joint inertia in our model. This braking behavior corresponds to "STOP 0 " of the robot. The

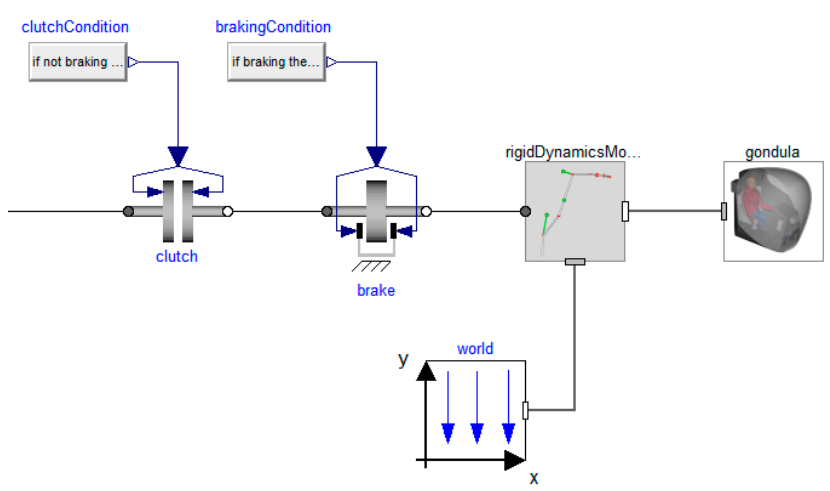

Fig. 2. The Modelica model of the simplified braking model. By coupling clutch and braking models from the Modelica standard library with a complete rigid dynamics model with friction and counterbalance unit, the braking behavior of the robot is simulated and the inputs for the HIC Criterion are generated.

values of the braking torques calculated during the simulation are equivalent to the evaluated braking behavior of the robot. Figure 3 illustrates an example of the end position of the experiment (left) and the simulation of the same trajectory (right). Nevertheless the acting acceleration at the TCP is very different because of the missing structural dynamics caused by stiffnesses and dampings of the robot, the cell and the dummys body.

\section{RESULTS}

For analyzing the resulting accelerations, an acceleration sensor is installed at the position of the pilot's head both in real experiments and simulations. For the simulation, the body is assumed to be totally fixed at the simulator cell and unable to move. The reason for not modeling the headneck-system is because of its complexity and the tremendous influence on the results. The outcome of this work is meant to give a tendency of the found WCBT. Thus no damping terms except of the counterbalance unit are modeled. These approximations lead to much higher accelerations at the head due to the fact that the whole kinetic energy has to be completely absorbed by the brakes.

For verification and comparison of the results, former experiments [6] are simulated with this new approach. Afterwards, WCBT are primarily simulated and evaluated. 


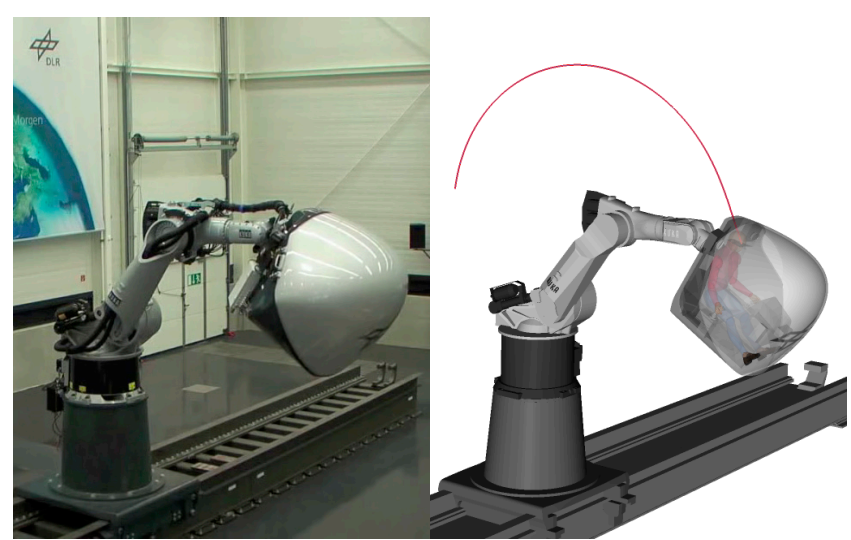

Fig. 3. Estimating the braking torques from experiments to simulate the robot behavior during emergency braking.

\section{A. Optimization setup}

The optimization problem is solved using the DLR Optimization Library for Modelica [14] using a sequential quadratic programming algorithm because of the nonlinearity of (6) and its capability of taking inequality constraints into account. Because of local maxima there is initially a rasterization of the workspace by systematic tuner variation done for the tuner parameters $q_{e}$ and $\dot{q}_{e}$. The maximum kinetic energy can only be reached if all joints move with their maximum possible velocities which in turn is not possible for every position because of the limiting motor torques.

The tuner variation needs discrete steps which are the minimum and maximum velocity for each joint, $\underline{\dot{q}}_{e}= \pm \beta \underline{\dot{q}}_{\max }$ and for $\underline{q}_{e} 5$ equidistant values from $\underline{q}_{\min , \alpha}$ to $\underline{q}_{\max , \alpha}$ for each joint.

The best result out of this first rasterization is taken as starting position for the sequential quadratic programming algorithm.

The values used as constraints are listed in table I.

\section{B. Comparison between former experiments and simulations}

To verify the model, experimental data of the whiplash maneuver is taken from [6] and the trajectory is reused for the simulation. The measured accelerations at the head of the pilot have been compared to the simulated accelerations. The commanded joint angles of axes 2, 3 and 5 are shown in figure 4. After the emergency stop, the behavior of the joint angles of the simulated robot during braking can be seen in figure 4 on the right side of the dashed line. The scaled acceleration of the experiment (see figure 5, upper diagram), and simulation (see figure 5, lower diagram), differ especially because of the missing movability of the dummy's body. The maximum scaled acceleration of the experiment was $5.33 \mathrm{~g}$ and for the simulation $6.97 \mathrm{~g}$. From this point on, we will use these results to compare them with the optimized simulations and to determine whether the generated trajectory could lead to a more dangerous situation in a real experiment, not to predict quantitative values of experiments.

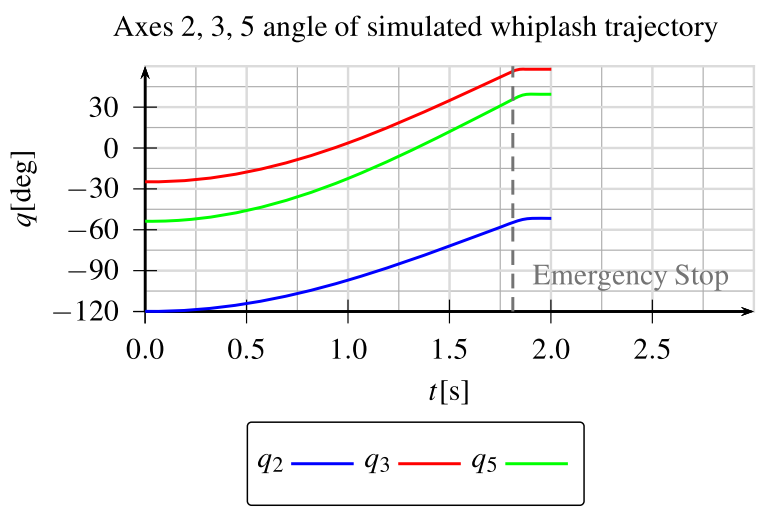

Fig. 4. Joint angles during the whiplash trajectory from [6]. The values after the emergency stop result from the simulated emergency braking model.

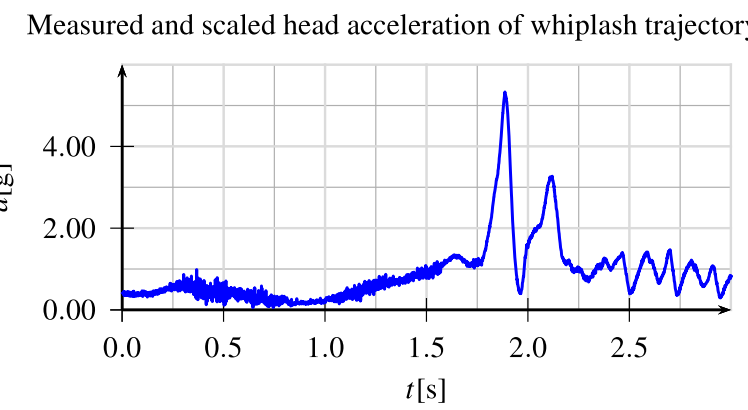

Simulated and scaled head acceleration of whiplash trajectory

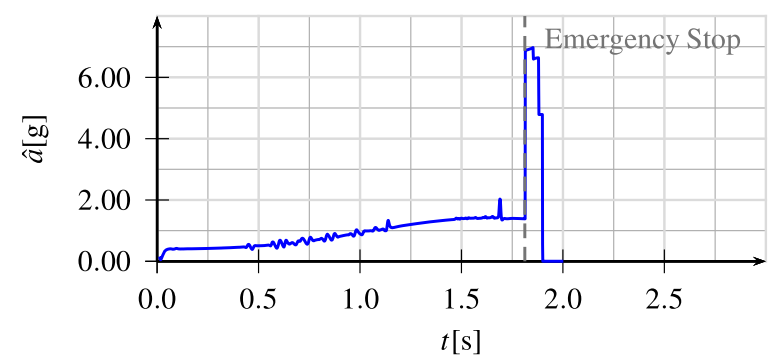

Fig. 5. Scaled head acceleration of the dummy's head sensors of the real whiplash experiment [6] (upper diagram) and scaled head acceleration during the simulated whiplash trajectory (lower diagram).

\section{Generation and simulation of WCBT}

The maximization of the kinetic energy with the constraints from table I and a load of $350 \mathrm{~kg}$ at the tool center point (TCP) lead to the WCBT as seen in figure 6 .

Figure 7 shows the position of the joints 1 to 7 . At $t=2.5 \mathrm{~s}$ the brakes become active and the clutches are released. The possible limits of the joint angles are not exploited. Using the presented method, the joint accelerations of the acceleration phase are at their maximum, which can be seen by the constantly increasing joint velocity (see figure 8 ). Figure 9 shows the percentage of the used scaled motor torques $\hat{\tau}=\tau / \tau_{\max }$. Because of its low dynamics, the linear axis defines the time for the whole trajectory $t_{\max }=2.5 \mathrm{~s}$, and thus is the first of the robot axes starting accelerating $\left(t_{s}=0\right)$. At the end configuration the kinetic energy of the head is maximized. 


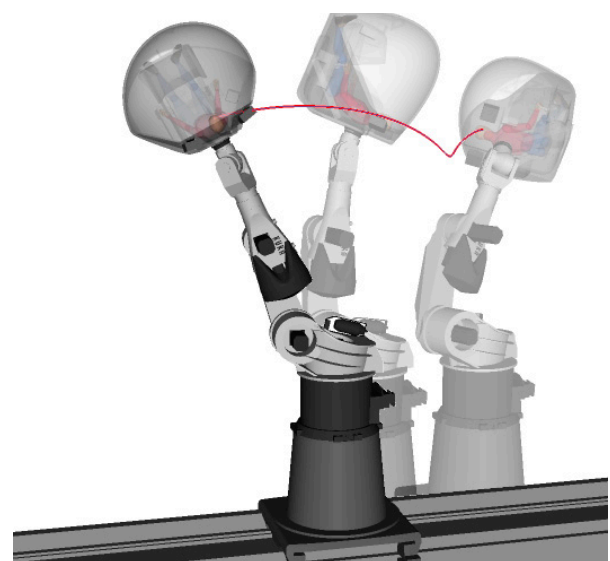

Fig. 6. Movement of the DLR Robotic Motion Simulator performing the optimized worst case braking trajectory.

Axes 1-6 angle of simulated worst case braking trajectory

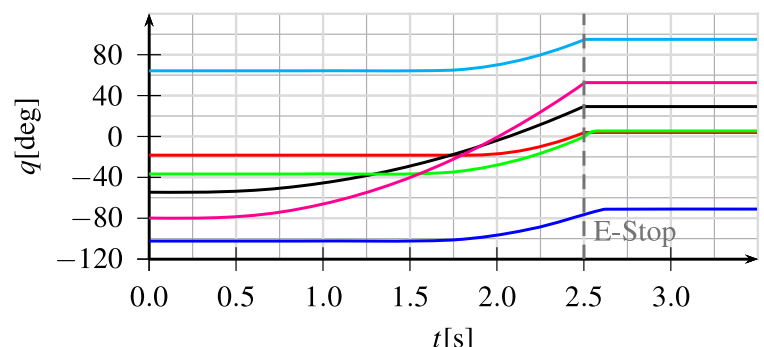

$$
q_{1}-q_{2}-q_{3}-q_{4}-q_{5}-q_{6}-
$$

Axis 7 position of simulated worst case braking trajectory

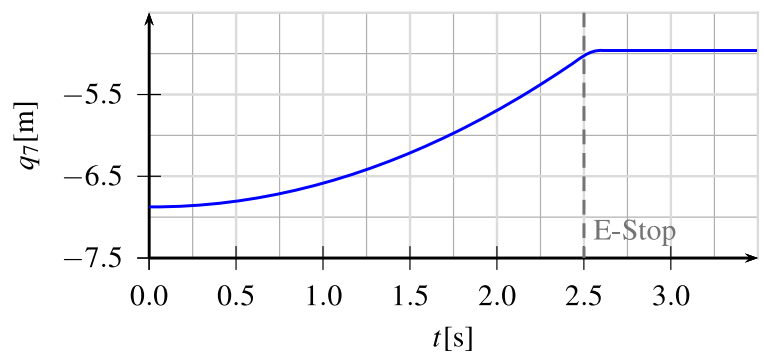

Fig. 7. Position of the robot axes during the worst case braking trajectory.

Changing the load at the TCP has only a weak influence on the trajectory. In comparison to the simulation of the unoptimized whiplash trajectory, the scaled head acceleration of the WCBT is 3 times higher, see figures 10 and 5 (lower diagram). Table III shows the HIC values and the maximum scaled acceleration of the experiments and the simulations. The HIC value for the real experiment was measured to be 2.34, the HIC value for the simulated whiplash is 4.52 . In comparison to this the HIC value of the WCBT increased to 14.87 , highlighting the importance of selecting worst case test trajectories for safety assessments.

As per [15], the lower limit for the $\mathrm{HIC}_{36}$ value considered threatening is 135 . Although the simulated trajectories lead to a HIC value that is well within the aforementioned threatening limit, these values are higher than the ones
Axes 1-6 velocity of simulated worst case braking trajectory

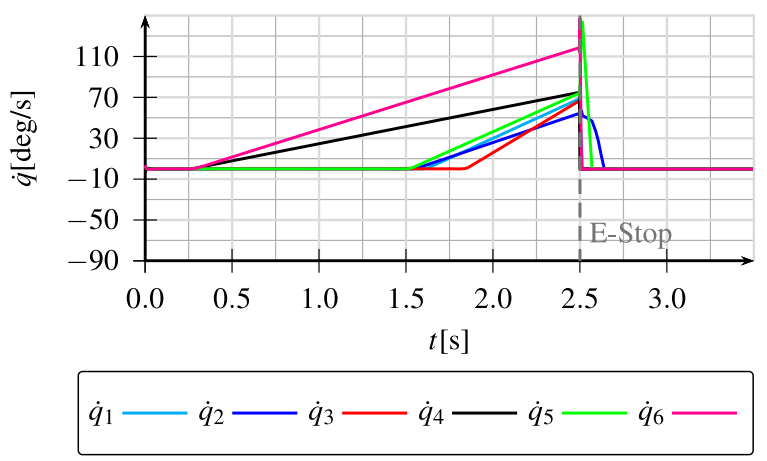

Axis 7 velocity of simulated worst case braking trajectory

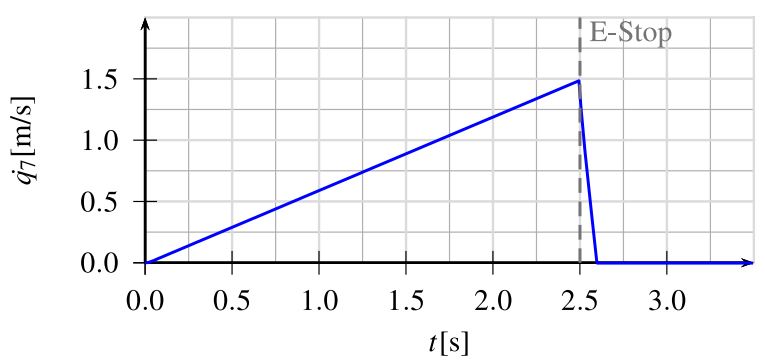

Fig. 8. Velocities of the robot axes during the worst case braking trajectory.

Normed motor torques of simulated worst case braking trajectory

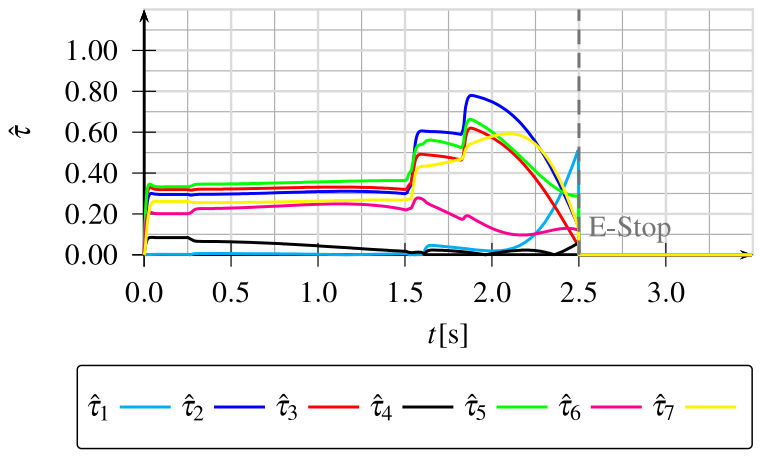

Fig. 9. Usage of motor torques normed to limits.

Simulated and scaled head acceleration of worst case braking trajectory

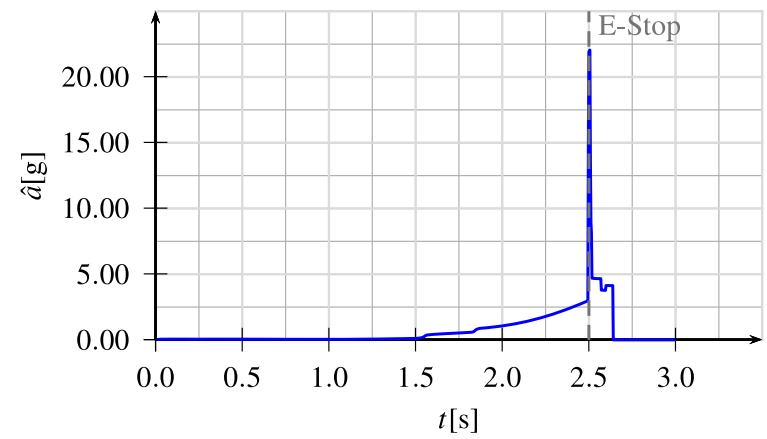

Fig. 10. Scaled acceleration of the worst case braking trajectory. 
observed during the tests in [6]. This can be attributed to missing dynamics of the head-neck system, the non-frontal direction of the acceleration vector, etc.

\section{CONCLUSION AND FUTURE WORK}

In this work, a method for the generation of optimized worst case braking trajectories (WCBT) for safety tests of robotic motion simulators has been presented. The simulation of the test results, based on a model for the emergency brakes, shows a considerable increase of the HIC value compared to heuristic trajectories like the whiplash trajectory from [6]. Because of the approximations assumed in the simulation model, it is necessary to validate these results with dummy tests on the real robot system to check for neglected effects like structural dynamics, the dynamics of the pilot's head and body in the simulator cell and the influence of the simplified braking model. In the next steps, the braking model should be extended and validated for the different stop modes. Finding the maximum kinetic energy is still a gradient based procedure and thus prone to local maxima. The rasterization minimizes the threat of getting stuck in a smaller local maxima but does not prevent it. By using heuristic optimization methods like particle swarm optimization or finer rasterization, the likelihood to find the global maximum could be improved.

In order to examine other dangerous braking maneuvers for example such leading to rotation around the yaw axis of the pilot's head, the optimization criterion can be changed easily to generate a trajectory leading to this dangerous motion.

\begin{tabular}{crrrrrr} 
Joint & \multicolumn{1}{c}{$q_{\min }$} & \multicolumn{1}{c}{$q_{\min , \alpha}$} & \multicolumn{1}{c}{$q_{\max , \alpha}$} & $q_{\max }$ & $\beta \dot{q}_{\max }$ & \multicolumn{1}{c}{$\gamma \ddot{q}_{\max }$} \\
\hline 1 & $-\infty^{\circ}$ & $-\infty^{\circ}$ & $+\infty^{\circ}$ & $+\infty^{\circ}$ & $70^{\circ} / \mathrm{s}$ & $78^{\circ} / \mathrm{s}^{2}$ \\
2 & $-128^{\circ}$ & $-110.8^{\circ}$ & $-59.2^{\circ}$ & $-42^{\circ}$ & $56^{\circ} / \mathrm{s}$ & $58^{\circ} / \mathrm{s}^{2}$ \\
3 & $-30^{\circ}$ & $-8.6^{\circ}$ & $55.6^{\circ}$ & $77^{\circ}$ & $69^{\circ} / \mathrm{s}$ & $102^{\circ} / \mathrm{s}^{2}$ \\
4 & $-90^{\circ}$ & $-54^{\circ}$ & $54^{\circ}$ & $90^{\circ}$ & $76^{\circ} / \mathrm{s}$ & $34^{\circ} / \mathrm{s}^{2}$ \\
5 & $-58^{\circ}$ & $-34.8^{\circ}$ & $34.8^{\circ}$ & $58^{\circ}$ & $76^{\circ} / \mathrm{s}$ & $76^{\circ} / \mathrm{s}^{2}$ \\
6 & $-90^{\circ}$ & $-54^{\circ}$ & $54^{\circ}$ & $90^{\circ}$ & $120^{\circ} / \mathrm{s}$ & $54 \% \mathrm{~s}^{2}$ \\
7 & $-9.4 \mathrm{~m}$ & $-7.6 \mathrm{~m}$ & $-2.2 \mathrm{~m}$ & $-0.4 \mathrm{~m}$ & $1.5 \mathrm{~m} / \mathrm{s}$ & $0.6 \mathrm{~m} / \mathrm{s}^{2}$ \\
& \multicolumn{5}{c}{ TABLE I }
\end{tabular}

JOINT LIMITS FOR OPTIMIZATION CONSTRAINTS.

\begin{tabular}{crrrrr} 
Joint & \multicolumn{1}{c}{$q_{s}$} & \multicolumn{1}{c}{$q_{e}$} & \multicolumn{1}{c}{$\dot{q}_{e}$} & \multicolumn{1}{c}{$\ddot{q}_{e}$} & \multicolumn{1}{c}{$t_{s}$} \\
\hline 1 & $79.9^{\circ}$ & $95.7^{\circ}$ & $69.9^{\circ} / \mathrm{s}$ & $78^{\circ} / \mathrm{s}^{2}$ & $1.59 \mathrm{~s}$ \\
2 & $-88.9^{\circ}$ & $-75.6^{\circ}$ & $55.6^{\circ} / \mathrm{s}$ & $58^{\circ} / \mathrm{s}^{2}$ & $1.54 \mathrm{~s}$ \\
3 & $-6.8^{\circ}$ & $4.8^{\circ}$ & $68.8^{\circ} \%$ & $102^{\circ} / \mathrm{s}^{2}$ & $1.83 \mathrm{~s}$ \\
4 & $-12.0^{\circ}$ & $30.5^{\circ}$ & $75.6^{\circ} \%$ & $34^{\circ} / \mathrm{s}^{2}$ & $0.25 \mathrm{~s}$ \\
5 & $-18.0^{\circ}$ & $0.55^{\circ}$ & $75.6^{\circ} / \mathrm{s}$ & $76^{\circ} / \mathrm{s}^{2}$ & $1.50 \mathrm{~s}$ \\
6 & $-13.0^{\circ}$ & $53.6^{\circ}$ & $119.7^{\circ} / \mathrm{s}$ & $54^{\circ} / \mathrm{s}^{2}$ & $0.27 \mathrm{~s}$ \\
7 & $-5.9 \mathrm{~m}$ & $-5 \mathrm{~m}$ & $1.5 \mathrm{~m} / \mathrm{s}$ & $0.6 \mathrm{~m} / \mathrm{s}^{2}$ & $0 \mathrm{~s}$ \\
\multicolumn{5}{c}{ TABLE II }
\end{tabular}

WORST CASE TRAJECTORY FOR THE DLR ROBOTIC MOTION SIMULATOR WITH $350 \mathrm{~kg}$ AT TCP.

\begin{tabular}{cccc} 
& $\begin{array}{c}\text { whiplash } \\
\text { experiment }\end{array}$ & $\begin{array}{c}\text { simulation } \\
\text { whiplash }\end{array}$ & WCBT \\
\hline $\mathrm{HIC}_{36}$ & 2.34 & 4.52 & 14.87 \\
$\hat{a}_{\text {max }}$ & 5.64 & 6.97 & 21.93
\end{tabular}

TABLE III

MAXIMUM ACCELERATIONS OF EXPERIMENT AND SIMULATIONS

\section{ACKNOWLEDGMENT}

We want to thank Johann Heindl, Miguel Neves, Sami Haddadin, Sebastian Minning, Sven Parusel and Tim Rokahr for their help with the first experiments leading to the motivation for this paper and the availability of real data.

\section{REFERENCES}

[1] T. Bellmann, M. Otter, and G. Hirzinger, "The DLR Robot Motion Simulator Part II: Optimization based path-planning," in Robotics and Automation (ICRA), 2011 IEEE International Conference on, 2011, pp. 4702-4709.

[2] M. Kleer, O. Hermanns, K. Dreßler, and S. Müller, "Driving simulations for commercial vehicles-a technical overview of a robot based approach," Proceedings of the driving simulation conference europe 2012,2012

[3] H. Teufel, H. Nusseck, K. Beykirch, J. Butler, M. Kerger, H. Bülthoff et al., "MPI Motion Simulator: Development and Analysis of a Novel Motion Simulator," in AIAA Modeling and Simulation Technologies Conference and Exhibit, 2007, pp. 1-11.

[4] T. Bellmann, J. Heindl, M. Hellerer, R. Kuchar, K. Sharma, and G. Hirzinger, "The DLR Robot Motion Simulator Part I: Design and Setup," in Robotics and Automation (ICRA), 2011 IEEE International Conference on, 2011, pp. 4694-4701.

[5] K. Sharma, S. Haddadin, J. Heindl, T. Bellmann, S. Parusel, T. Rokahr, S. Minning, and G. Hirzinger, "Serial kinematics based motion simulator - evaluation of safety of the passenger," in The International Conference on the Safety of Industrial Automated Systems (SIAS), 2012.

[6] K. Sharma, S. Haddadin, S. Minning, J. Heindl, T. Bellmann, S. Parusel, T. Rokahr, and A. Albu-Schaeffer, "Evaluation of Human Safety in the DLR Robotic Motion Simulator using a Crash Test Dummy," in Robotics and Automation (ICRA), 2013 IEEE International Conference on, 2013.

[7] K. H. Digges, "Injury Measurements and Criteria," Models for Aircrew Safety Assessment: Uses, Limitations and Requirements, 1999.

[8] S. Haddadin, A. Albu-Schäffer, and G. Hirzinger, "Safety Evaluation of Physical Human-Robot Interaction via Crash-Testing," in Robotics: Science and Systems, 2007.

[9] J. Versace, "A Review of the Severity Index," in 15th Stapp Car Crash Conference Proceedings, 15th Stapp Car Crash Conference Proceedings, Ed., 1971, pp. 771-796.

[10] Assemessment Protocol - Adult Occupant Protection, EuroNCAP, January 2013. [Online]. Available: http://www.euroncap.com/files/Euro-NCAP-Assessment-ProtocolAOP-v5.5-0-c9cbb083-b4e6-4788-8496-81394a8788a7.pdf

[11] O. Bostroem, M. Y. Svensson, B. Aldman, H. A. Hansson, Y. Haaland, P. Loevsund, T. Seeman, A. Suneson, A. Saeljoe, and T. Oertengren, "A new neck injury criterion candidate-based on injury findings in the cervical spinal ganglia after experimental neck extension trauma," in Proceedings Of The 1996 International Conference On The Biomechanics Of Impact (IRCOBI), Dublin, Ireland, no. 00767056, 1996, pp. 123-36.

[12] M. V. Mark W. Spong, Seth Hutchingson, Robot Modeling and Control. John Wiley \& Sons, 2006.

[13] KUKA KR 360-3; KR 500-3 Specification, online, KUKA Roboter GmbH. [Online]. Available: http://www.kuka-robotics.com/res/sps/e6c77545-9030-49b193f5-4d17c92173aa_Spez_KR_360-3_KR_500-3_en.pdf

[14] A. Pfeiffer, "Optimization Library for Interactive Multi-Criteria Optimization Tasks," in 9th International Modelica Conference, 2012.

[15] A. Patel and T. Goswami, "Comparison of Intracranial Pressure by Lateral and Frontal Impacts-Validation of Computational Model," 2012. 